\title{
Jones formalism for image fusion
}

\author{
${ }^{1}$ Khaustov D. Ye., ${ }^{1}$ Khaustov Ya. Ye., ${ }^{1}$ Ryzhov Ye., ${ }^{2}$ Lychkowskyy E., \\ ${ }^{3}$ Vlokh R. and ${ }^{1}$ Nastishin Yu. A. \\ ${ }^{1}$ Hetman Petro Sahaidachnyi National Army Academy, 32 Heroes of Maidan Street, \\ 79012 Lviv, Ukraine \\ ${ }^{2}$ Lviv Danylo Halytsky National Medical University, 69 Pekarska Street, 79010 \\ Lviv, Ukraine \\ ${ }^{3}$ O. G. Vlokh Institute of Physical Optics, 23 Dragomanov Street, 79005 Lviv, \\ Ukraine
}

Received: 08.07 .2021

\begin{abstract}
We suggest a novel approach for the fusion of visible $(u)$ and infrared $(v)$ images, basing on analogy between the mathematical forms of a Jones vector of elliptically polarized light wave and a complex 2D vector $\vec{\psi}^{0}$ composed of the images $u$ and $v$. Since there is no restriction on which of the two images should be chosen as a real (or imaginary) component, one can construct $\vec{\psi}^{0}$ in the two forms, $\vec{\psi}_{\text {neg }}^{0}=(1 / \sqrt{2})[u, i v]^{T r} \quad$ or $\quad \vec{\psi}_{\text {pos }}^{0}=(1 / \sqrt{2})[v, i u]^{T r}$, where the superscript "Tr" denotes the operation of transposing, i.e. $\vec{\psi}^{0}$ represents a column vector. Following the analogy with the Jones vector of light wave, the vector $\vec{\psi}_{\text {pos, neg }}^{0}$ can be transformed as $\vec{\psi}=J \vec{\psi}_{n e g}^{0}$, pos , with $J$ being a complex $2 \times 2$-matrix, an analogue of the Jones matrix for optically anisotropic medium. The above analogy with the Jones formalism allows one to synthesize the fused images using three types of the fusion algorithms, 'amplitude', 'azimuth' and 'ellipticity' ones. Varying the components of the $J$ matrix with time, one can synthesize the fused image in a dynamic mode, thus animating the images fused under smoothly varying parameters, which are combinations of the $J$ matrix components.
\end{abstract}

Keywords: image fusion, Jones matrices, complex Jones vectors, visible and infrared images

UDC: 004.932

\section{Introduction}

Fusion of images is one of plentiful benefits of digital imaging in comparison with traditional analog photography based on photo-chemical developing process, at which an image is printed on a solid substrate (paper, polymer, textile, etc.). A digital image is recorded in the form of a computer file containing a brightness table of pixels. The essence of image fusion is to combine the brightness tables of two partial images of the same scene. This fused image enriches the amount of information carried by the two partial images.

The brightness tables of partial images can be fused either on the lowest spatial image level by applying conventional arithmetic or logic operations to the pairs of pixels of the two partial brightness tables - or by operating with the tables on a super-pixel level and applying specially developed sub-space and multi-scale fusion techniques [1]. Nowadays sophisticated imageprocessing procedures [2-6], deep learning [7-9] and neural-network approaches [10-12] are 
employed for the image fusion. As a result, the pixel-level techniques based on simple arithmetic algorithms might seem to be rather primitive and only of educational interest. However, it turns out that the actual situation is different. The arithmetic pixel-fusion techniques still remain popular, and we believe that their development will be permanently demanded, at least, in the nearest years. The reasons are as follows. While the highly intellectual modern super-pixel fusion techniques are designed for the teams of qualified users who work in a computer office equipped with modern high-power computing facilities, the arithmetic pixel-fusion approaches represent an express-technique which is preferable when a real-time, out-of-office image fusion should be done in extreme situations with small-size mobile imaging devices by the users who have no special skills in programming.

Although many practical examples of the multimodal imaging are concerned with the visible (Vis) and infrared (IR) spectral ranges, there is also a great need in this approach for any electromagnetic ranges (i.e., $\gamma$-, $\mathrm{X}$-, ultraviolet, Vis, IR and radio-waves). The examples of the appropriate portable devices are numerous: portative imaging devices for medical diagnostics, target-sightseeing complexes for armoured vehicles, submarines, ships or spacecrafts (note that the size of an additional device does matter in the last case), compact low-weight smart imaging cameras for expeditions, etc.

In spite of the obvious needs, the list of available arithmetic pixel-fusion express-techniques is short, much shorter than that of the super-pixel fusion techniques. One of the reasons for this circumstance is that the information on the express-fusion techniques of military or other specialized sectors might be restricted. Another explanation is related to the very principle of arithmetic pixel fusion. Within the field of real scalars, it is limited by the four arithmetic operations (addition, subtraction, multiplication and division). Although there are the pixel-fusion techniques based on different principles (e.g., expectation-maximization or Markov random-field algorithms [1]), the latter are not considered as express-techniques because of their complexity. One of the possibilities for boosting novel rapid arithmetic pixel-fusion techniques is overstepping the limits of the field of real scalars and passing into the fields of complex scalars and vectors [13, 14].

Recently we have offered a complex scalar function as a template for the fusion of Vis and IR images $[13,14]$. In particular, we have generalized the fusion formalism from the field of complex scalar numbers to the field of complex vectors, such that one of the partial input images (Vis or IR) is chosen as a real component and the other image is an imaginary component of a complex $2 \mathrm{D}$ vector $\vec{\psi}_{0}$, which is uniquely defined by these components in a vector basis. Now we argue that, by its form, the complex vector $\vec{\psi}^{(0)}$ in this case is a formal analogue of the Jones vector describing the electric field of elliptically polarized light wave [15-17]. Then the image fusion resembles a superposition of eigenwaves in an optically anisotropic medium. Consequently, using the analogy with the Jones formalism, one can obtain a transformed fused image described by a vector $\vec{\psi}=J \vec{\psi}^{(0)}$, where $J$ is a $2 \times 2$-matrix consisting of complex components. Therefore the conclusions that follow from the mathematical properties of Jones-matrix transformations can be transferred onto the operations of image fusion.

The present article is organized as follows. Section 2 introduces the principles of our complex vector-image fusion (CVIF) method and the arguments supporting the analogy between the Jones formalism and the image fusion. Applications of the Jones-matrix transformations to the image fusion are considered in Section 3 and advantages of the CVIF method are discussed in Section 4. Finally, Section 5 concludes our results. 


\section{CVIF method}

Let Vis $(u)$ and IR $(v)$ images be components of a complex 2D vector $\vec{\psi}^{(0)}$, so that one of these images is a real component and the other image is an imaginary component of $\vec{\psi}^{0}$. Since there is no restriction on which of the two images must be real (or imaginary) component of the complex vector, one can construct two different complex vectors $\vec{\psi}_{n e g}^{(0)}$,pos for the same partial images $u$ and $v$ :

$$
\begin{aligned}
& \vec{\psi}_{\text {neg }}^{(0)}=\frac{1}{\sqrt{2}}\left[\begin{array}{c}
u \\
i v
\end{array}\right], \\
& \vec{\psi}_{\text {pos }}^{(0)}=\frac{1}{\sqrt{2}}\left[\begin{array}{c}
v \\
i u
\end{array}\right] .
\end{aligned}
$$

In the orthogonal basis of mutually orthogonal unit vectors $\vec{c}_{1}$ and $\vec{c}_{2}$, the complex vectors $\vec{\psi}_{\text {neg,pos }}^{(0)}$ can be represented as

$$
\begin{aligned}
& \vec{\psi}_{\text {neg }}^{(0)}=\frac{1}{\sqrt{2}}\left(u \vec{c}_{1}+i v \vec{c}_{2}\right), \\
& \vec{\psi}_{\text {pos }}^{(0)}=\frac{1}{\sqrt{2}}\left(v \vec{c}_{1}+i u \vec{c}_{2}\right) .
\end{aligned}
$$

Throughout the text, the notation $f_{\text {neg, pos }}$ for the parameter $f$ under consideration is a short form for the expression " $f_{\text {neg }}$ and/or $f_{\text {pos }}$ ", e.g. $\vec{\psi}_{\text {neg,pos }}^{(0)}$ stands for $\vec{\psi}_{\text {neg }}^{0}$ and $\vec{\psi}_{\text {pos }}^{(0)}$.

\subsection{Analogy of the fused image vector with the Jones vector of polarized light wave}

The forms of the complex vectors $\vec{\psi}_{\text {neg,pos }}^{(0)}$ given by Eqs. (1)-(4) bear a striking resemblance to the form of a Jones vector describing an elliptically polarized light wave [15-17]:

$$
\vec{E}=\frac{1}{\sqrt{2}}\left[\begin{array}{c}
E_{1} \\
i E_{2}
\end{array}\right],
$$

with the partial Vis $(u)$ and IR ( $v)$ images in Eqs. (1) and (2) corresponding to the electric-field components $E_{1}$ and $E_{2}$ of the Jones vector $\vec{E}$ in Eq. (5). We argue below that, in addition to the argument of explicit similarity of the mathematical forms given by Eqs. (1), (2) and (5), there are also important physical reasons to employ this analogy for the fusion of multimodal images. The arguments supporting the analogy between the Jones formalism and the fusion of Vis and IR images are as follows.

\subsubsection{Arguments for the analogy with Jones formalism?}

First, both the components $E_{1}$ and $E_{2}$ of the Jones vector in Eq. (5), on the one hand, and the parameters $u$ and $v$ of the partial Vis and IR images in Eqs. (1) and (2), on the other hand, vary between 0 and 1 .

Second, in the framework of Jones formalism, the light-wave components $E_{1}$ and $E_{2}$ correspond in general to two different eigenwaves propagating in an anisotropic medium (e.g., ordinary and extraordinary waves in an optically uniaxial transparent crystal [18]). The light wave $\vec{E}$ emerging from the medium is a result of superposition of the eigenwaves $\vec{E}_{1}=(1 / \sqrt{2}) E_{1} \vec{c}_{1}$ and 
$\vec{E}_{2}=(1 / \sqrt{2}) E_{2} \vec{c}_{2}$, where $\vec{c}_{1}$ and $\vec{c}_{2}$ are the unit vectors in the vector basis on which the Jones vectors are defined. Similarly, the complex vector $\vec{\psi}_{n e g, p o s}^{(0)}$ represents a result of fusion of the partial vector images $\vec{u}=(1 / \sqrt{2}) u \vec{c}_{1}$ and $\vec{v}=(1 / \sqrt{2}) v \vec{c}_{2}$. In other words, here the operation of image fusion is an analogue of the phenomenon of superposition of optical eigenwaves propagating in an optically anisotropic medium.

Third, $\vec{E}_{1}$ and $\vec{E}_{2}$ are independent vectors that correspond to different eigenwaves with different polarizations. As a result, they do not add as scalars, being superposed as complex vectors instead. Similarly, the two partial Vis $(u)$ and IR $(v)$ images are independent in the sense that they cannot be transformed into each other by any known image-processing procedure. In this context, it is worth recalling that the partial Vis and IR images differ by the physical principles of their recording. Moreover, as a rule, an object of interest (a target) appears to have the opposite contrasts on the Vis and IR images of the same scene. A Vis image of a target results from the interplay between the absorption and reflection of external light. Consequently, a typical target looks dark against a bright background on the Vis image (a positive local contrast). In the IR spectral range, a target usually emits IR waves and, therefore, its image results from the interplay between the light emission and reflection. Consequently, the same target usually looks bright against a dark background on the IR image (a negative contrast).

As a consequence, scalar summation of the partial images $u$ and $v$ would lead to significant lowering or even vanishing of the contrast of target in the fused image [19]. For this reason, the image fusion based on the addition in the field of real scalars is, at least, not desirable for the multimodal partial images [13, 14]. A transition to the complex scalar field solves the mentioned problem of vanishing contrast. We have shown in Refs. $[13,14]$ that the contrast doubles for the phase image obtained with a so-called complex scalar-function image fusion (SCFIF) method. On the contrary, the contrast becomes zero (i.e., a target becomes invisible) in the frame of the scalaraddition fusion method whenever the target has the contrasts which are equal in the absolute values but opposite in the signs in its partial Vis and IR images [19]. The same advantage is preserved when we pass to the complex-vector field in the framework of our CVIF method. Similarly to the phenomenon of superposition of the eigenwaves, which have different polarizations, the fusion of the multimodal images, which reveal the opposite contrasts, should also be performed in the field of complex vectors. The sign of the local contrast in the image, therefore, corresponds to the polarization of the eigenwaves within the suggested analogy. The opposite contrasts in the Vis and IR ranges correspond to the orthogonal polarizations of the optical eigenwaves.

Forth, the squared Jones-vector amplitude is the intensity $I$ of the wave, $I=|\vec{E}|^{2}=\vec{E} \cdot \vec{E}^{*}=\left(E_{1}^{2}+E_{2}^{2}\right) / 2$. Notice that, since the intensity of the wave is not necessarily equal to unity, the eigenwaves under consideration are orthogonal but not necessarily orthonormal. Under the condition $E_{2}=0$, the Jones vector describes the light wave linearly polarized along the axis "1" of the Cartesian coordinate system. Similarly, the light wave is linearly polarized along the coordinate axis "2" at $E_{1}=0$. Finally, the light wave is elliptically polarized in the general case $E_{1} \neq 0, E_{2} \neq 0$, and it becomes circularly polarized when we have the equality $E_{1}=E_{2}$. 
Quite similarly, the squared amplitude $\left|\psi_{\text {neg, pos }}^{(0)}\right|^{2}$ is the intensity of the image fused by the vector-amplitude algorithm. Under alternative conditions $v=0$ or $u=0$, the complex vector $\vec{\psi}_{\text {neg,pos }}^{(0)}$ of the fused image is reduced respectively to one of the partial (Vis or IR) images, which are analogues of the linearly polarized eigenwaves. At all the other $v$ and $u$ values, the fused complex-vector images $\vec{\psi}_{n e g, p o s}^{(0)}$ are analogues of the elliptically polarized waves, with the limiting case $u=v$ corresponding to the circularly polarized light wave. The independence of the $v$ and $u$ parameters implies that the vectors $\vec{u}=(1 / \sqrt{2}) u \vec{c}_{1}$ and $\vec{v}=(1 / \sqrt{2}) v \vec{c}_{2}$ of the partial images are orthogonal but not necessarily orthonormal.

The correspondence between the basic notions of the Jones formalism and the fusing CVIF technique, which has been discussed above, are summarized in Table 1.

Table 1. Analogies between the Jones formalism and the CVIF method

\begin{tabular}{l|l}
\hline \multicolumn{1}{c|}{ Jones formalism } & \multicolumn{1}{c}{ CVIF method } \\
\hline $\begin{array}{l}\text { Jones vector components, i.e. linearly } \\
\text { polarized eigenwaves } E_{1} \text { and } E_{2} \\
\text { Superposition of light eigenwaves }\end{array}$ & Partial Vis $(u)$ and IR $(v)$ images \\
$\begin{array}{l}\text { Jones vector } \vec{E} \text { of an elliptically } \\
\text { polarized light wave }\end{array}$ & Complex vector $\vec{\psi}_{\text {neg,pos }}^{(0)}$ of a fused image \\
$\begin{array}{l}\text { Polarization of light eigenwave } \\
\text { Intensity of light wave }|\vec{E}|^{2}, \text { squared }\end{array}$ & Sign of local contrast in partial image \\
amplitude of Jones vector & $\begin{array}{l}\text { Intity of fused vector image }\left|\vec{\psi}_{\text {neg,pos }}^{(0)}\right|^{2}, \text { squared } \\
\text { Azimuth of polarization ellipse }\end{array}$ \\
Ellipticity of polarization ellipse & $\begin{array}{l}\text { Ellipticity algorithm for fusing images, a measure of } \\
\text { relative contributions of partial images to a fused image }\end{array}$ \\
\hline
\end{tabular}

\subsubsection{What do we gain from the analogy?}

Analogies in the description of different physical phenomena are an efficient tool for deeper understanding of known and prediction of new properties and effects. An analogy between different physical systems can be based on the similarities found either in their structures and behaviours or in mathematical forms of equations that describe these systems. The structural element serves as a basis for analogy, e.g., between the solar system and the atom (a planetary model of atom). The same concerns the analogy between a freely suspended cholesteric liquidcrystal droplet with a tangential surface anchoring [20] and a magnetic Dirac monopole [21]. Maxwell has used behavioural similarity between the fluid and the electrical current in order to develop the theory for various fields of electricity [22, 23]. A similar approach has once been employed for the phenomena of superconductivity and superfluidity [24]. Following from similarity of the equations for the free energies of a superconductor and a smectic liquid crystal, de Gennes [25] has concluded that many effects, which occur in superconductors, should have their counterparts in liquid-crystal smectics A. In particular, unwinding of the cholesteric twist at the transition into smectic-A phase has been understood as a Meisner effect for superconductor in magnetic field, whereas the twist grain-boundary phases in chiral liquid crystals have been 
predicted [26] and discovered experimentally [27-30] basing on the analogy with the Abrikosov phase of superconductor in magnetic field.

This is why a similarity between the superposition of electromagnetic waves with different polarizations and the fusion of multimodal images has inspired us to assign the complex vector $\vec{\psi}_{n e g, p o s}^{(0)}$ to a fused image built of partial Vis and IR images, such that the $\vec{\psi}_{n e g}^{(0)}$,pos parameter given by Eqs. (1) and (2) is an analogue of the Jones vector (see Eq. (5)) for elliptically polarized wave. As explained below, the Jones formalism opens an efficient way for describing transformations of the vector $\vec{\psi}_{n e g}^{(0)}$,pos under the action of a Jones matrix.

In the framework of Jones formalism, a Jones vector $\vec{E}^{(0)}$ transforms into another vector $\vec{E}=J \vec{E}^{(0)}$ under the action of a $2 \times 2$-Jones matrix $J$, which has complex components. Because the four components of the Jones matrix are complex, they contain eight independent parameters together. In the most general case, the Jones matrix $J$ describes the anisotropic medium possessing eight different optical properties. These are refraction, absorption, linear birefringence, linear dichroism, circular birefringence, circular dichroism, Jones birefringence, and Jones dichroism. Each of these eight parameters affects in a specific manner the components of Jones vector. This modifies the following parameters describing the optical wave: the intensity (amplitude), the azimuth, and the ellipticity of polarization ellipse. Since the transformation of the electric-field vector of optical wave under the action of each of these parameters is well understood and can be easily predicted, the analogy of the fused-image vector $\vec{\psi}$ with the Jones vector can be fruitfully employed when fusing images according to the well-known rules of the Jones-matrix formalism. This does not require recalculating their action on the components of the complex vector $\vec{\psi}_{0}$.

Using an analogous Jones transformation, one can obtain a vector $\vec{\psi}_{n e g, p o s}=J \vec{\psi}_{n e g, p o s}^{(0)}$. In the same way as the Jones-matrix components affect the components of the input Jones vector, they affect the input partial images ( $u$ and $v$ ). Then the analogues of the parameters such as the intensity (or the amplitude), the azimuth and the ellipticity can be calculated for the transformed vector $\vec{\psi}_{n e g, p o s}$. The latter can be used for inventing three different fusion algorithms, each of which carries its own information.

The polarization states of optical waves can be represented graphically with a standard Poincare sphere. Then a possibility for graphical representation of the evolution of transformed fused vector $\vec{\psi}_{\text {neg,pos }}$ will represent another benefit of the analogy between the fusion and the Jones formalism. The coordinates of different points on the Poincare sphere are specified by the azimuth and ellipticity angles, while the radius of this sphere corresponds to the degree of polarization of optical wave. An analogous parameter can be calculated for the fused vector $\vec{\psi}_{\text {neg,pos }}$. Importantly, the data obtained from the fused vector $\vec{\psi}_{\text {neg,pos }}$ as an analogue of the Jones vector can be used to calculate the analogue of the Stokes vector. Then the Mueller-matrix formalism can be applied to the image fusion.

In some cases the matrix $J$ can be reduced to the Jones matrices of particular optical elements such as linear and circular polarizers, linear phase-retardation plates (e.g., quarter- or full-wave ones), absorbing or dichroic plates, optical rotators, etc. The effects of these optical 
elements on the light wave are well understood and, hence, their analogues can be efficiently applied to the fusion vector $\vec{\psi}_{n e g, p o s}$. In this way, the corresponding calculations can be performed according to well-known rules and with well predictable results. Moreover, additional possibilities for graphical consideration would appear basing on the Poincare sphere.

\subsubsection{Parameters of $\vec{\psi}_{\text {neg,pos }}$ vector as a basis for fusion algorithms}

Using the analogy with the Jones vector of light wave, the vectors $\vec{\psi}_{n e g, p o s}$ can be characterized by the amplitude $\left|\psi_{\text {neg,pos }}^{(0)}\right|$, as well as by the azimuth $\chi_{\text {neg,pos }}^{(0)}$ and the ellipticity angle $\gamma_{\text {neg,pos }}^{(0)}$ of polarization ellipse. These points can serve as origin of three different types of fusion algorithms, which we call as amplitude, azimuth and ellipticity algorithms. It is easy to show that the both vectors $\vec{\psi}_{\text {neg }}^{(0)}$ and $\vec{\psi}_{\text {pos }}^{(0)}$ have the same amplitude,

$$
\left|\vec{\psi}^{(0)}\right|=\left|\vec{\psi}_{\text {neg }}^{(0)}\right|=\sqrt{\left(\vec{\psi}_{\text {neg }}^{(0)}\right) \cdot\left(\vec{\psi}_{\text {neg }}^{(0)}\right)^{*}}=\left|\vec{\psi}_{\text {pos }}^{(0)}\right|=\sqrt{\left(\vec{\psi}_{\text {pos }}^{(0)}\right) \cdot\left(\vec{\psi}_{\text {pos }}^{(0)}\right)^{*}}=\frac{1}{\sqrt{2}} \sqrt{u^{2}+v^{2}},
$$

where the symbols ". "and "*" denote the scalar product and the complex conjugate, respectively. The azimuth $\chi$ and the ellipticity $\gamma$ are defined by the relations [21]

$$
\begin{aligned}
& \tan 2 \chi_{\text {neg }, p o s}^{(0)}=\frac{t_{\text {neg, }, \text { pos }}^{(0)}+\left(t_{\text {neg }, p o s}^{(0)}\right)^{*}}{1-\left|t_{\text {neg }, p o s}^{(0)}\right|}, \\
& \sin 2 \gamma_{\text {neg }, p o s}^{(0)}=-i \frac{t_{\text {neg, pos }}^{(0)}-\left(t_{\text {neg }, p o s}^{(0)}\right)^{*}}{1+\left|t_{\text {neg }, p o s}^{(0)}\right|},
\end{aligned}
$$

where

$$
t_{\text {neg }}^{(0)}=i \frac{v}{u},\left|t_{\text {neg }}^{(0)}\right|=\sqrt{t_{\text {neg }}^{(0)}\left(t_{\text {neg }}^{(0)}\right)^{*}}=\frac{v}{u}, \quad t_{\text {pos }}^{(0)}=i \frac{u}{v}, \quad\left|t_{\text {pos }}^{(0)}\right|=\sqrt{t_{\text {pos }}^{(0)}\left(t_{\text {pos }}^{(0)}\right)^{*}}=\frac{u}{v} .
$$

It is worth noting that, in the framework of the SCFIF method [14], $\left|\vec{\psi}^{(0)}\right|=(1 / \sqrt{2}) \sqrt{u^{2}+v^{2}}$ implies nothing but the amplitude algorithm (see Eq. (5) in Ref. [14]), whereas $\left|t_{\text {neg }}^{(0)}\right|=\frac{v}{u}$ and $\left|t_{\text {pos }}^{(0)}\right|=\frac{u}{v}$ correspond to the $t$-algorithms of the SCFIF method (see Eqs. (6) and (7) in Ref. [14]).

Substitution of Eqs. (9) in Eqs. (7) and (8) gives

$$
\begin{gathered}
\chi^{(0)}=\chi_{\text {neg }}^{(0)}=\chi_{\text {pos }}^{(0)}=0, \\
\sin 2 \gamma^{(0)}=\sin 2 \gamma_{\text {neg }}^{(0)}=\sin 2 \gamma_{\text {pos }}^{(0)}=2 \frac{u v}{u^{2}+v^{2}} .
\end{gathered}
$$

The other functions of the ellipticity angle might also be useful as fusion algorithms:

$$
\begin{aligned}
& \cos 2 \gamma^{(0)}=\sqrt{\left(\frac{u^{2}-v^{2}}{u^{2}+v^{2}}\right)^{2}}=\frac{\left|u^{2}-v^{2}\right|}{u^{2}+v^{2}}, \\
& \tan 2 \gamma^{(0)}=2 \frac{u v}{\sqrt{\left(u^{2}-v^{2}\right)^{2}}}=2 \frac{u v}{\left|u^{2}-v^{2}\right|} .
\end{aligned}
$$


Notice that Eq. (11) remains the same when $u^{(0)}$ and $v^{(0)}$ are interchanged, thereby implying the equality $\gamma_{\text {neg }}^{(0)}=\gamma_{\text {pos }}^{(0)}$. Consequently, the same holds for Eqs. (12) and (13).

\section{Jones matrix approach for image fusion}

Consider a matrix

$$
J=\left[\begin{array}{ll}
J_{11} & J_{12} \\
J_{21} & J_{22}
\end{array}\right]=\left[\begin{array}{ll}
J_{11}^{\prime}+i J_{11}^{\prime \prime} & J_{12}^{\prime}+i J_{12}^{\prime \prime} \\
J_{21}^{\prime}+i J_{21}^{\prime \prime} & J_{22}^{\prime}+i J_{22}^{\prime \prime}
\end{array}\right] .
$$

The matrix $J$ transforms the vectors $\vec{\psi}_{\text {neg }}^{(0)}$ and $\vec{\psi}_{\text {pos }}^{(0)}$ into the vectors

such that we obtain

$$
\vec{\psi}_{n e g, p o s}=J \vec{\psi}_{n e g, p o s}^{(0)},
$$

$$
\vec{\psi}_{\text {neg }}=\left|\begin{array}{l}
\left(J_{11}^{\prime} u-J_{12}^{\prime \prime} v\right)+i\left(J_{11}^{\prime \prime} u+J_{12}^{\prime} v\right) \\
\left(J_{21}^{\prime} u-J_{22}^{\prime \prime} v\right)+i\left(J_{21}^{\prime \prime} u+J_{22}^{\prime} v\right)
\end{array}\right|, \quad \vec{\psi}_{p o s}=\left|\begin{array}{l}
\left(J_{11}^{\prime} v-J_{12}^{\prime \prime} u\right)+i\left(J_{11}^{\prime \prime} v+J_{12}^{\prime} u\right) \\
\left(J_{21}^{\prime} v-J_{22}^{\prime \prime} u\right)+i\left(J_{21}^{\prime \prime} v+J_{22}^{\prime} u\right)
\end{array}\right| .
$$

\subsection{Amplitude algorithms}

The amplitudes of the transformed vectors $\vec{\psi}_{\text {neg }}$ and $\vec{\psi}_{\text {pos }}$ read as

where

$$
\left|\vec{\psi}_{n e g, p o s}\right|^{2}=\left(a_{n e g, p o s}^{\psi} u\right)^{2}+\left(b_{n e g, p o s}^{\psi} v\right)^{2}+\left(c_{n e g, p o s}^{\psi}\right)^{2} u v,
$$

$$
\begin{aligned}
& \left(a_{\text {neg }}^{\psi}\right)^{2}=\left(b_{\text {pos }}^{\psi}\right)^{2}=\left|J_{11}\right|^{2}+\left|J_{21}\right|^{2}, \\
& \left(b_{\text {neg }}^{\psi}\right)^{2}=\left(a_{\text {pos }}^{\psi}\right)^{2}=\left|J_{22}\right|^{2}+\left|J_{12}\right|^{2}, \\
& \left(c_{\text {neg }}^{\psi}\right)^{2}=\left(c_{\text {pos }}^{\psi}\right)^{2}=2\left(J_{11}^{\prime \prime} J_{12}^{\prime}-J_{11}^{\prime} J_{12}^{\prime \prime}+J_{22}^{\prime} J_{21}^{\prime \prime}-J_{22}^{\prime \prime} J_{21}^{\prime}\right) .
\end{aligned}
$$

Eq. (17) shows that, in general, the inequality

$$
\left|\vec{\psi}_{\text {neg }}\right| \neq\left|\vec{\psi}_{\text {pos }}\right|
$$

holds true, which is different from the corresponding algorithm used in the framework of the scalar SCFIF method [13,14]. It is also different from the amplitude $\left|\vec{\psi}^{(0)}\right|$ (see Eq. (6)). Therefore, one can obtain two different amplitude images $\left|\vec{\psi}_{n e g}\right|$ and $\left|\vec{\psi}_{p o s}\right|$ after the action of the $J$ matrix, instead of one amplitude image.

Taking into account that the amplitudes $\left|\vec{\psi}_{\text {neg,pos }}\right|$ represent some fused images, one should normalize their maximal possible value by imposing the condition

$$
\left(\left|\vec{\psi}_{\text {neg, pos }}\right|_{\max }\right)^{2}=1 \text {. }
$$

Substitution of the maximal possible values of the partial images $\left(u_{\max }^{(0)}=1\right.$ and $\left.v_{\max }^{(0)}=1\right)$ into Eq. (20) gives

$$
\left(a_{\text {neg, }, \text { pos }}^{\psi}\right)^{2}+\left(b_{\text {neg, pos }}^{\psi}\right)^{2}+\left(c_{\text {neg, pos }}^{\psi}\right)^{2}=1 .
$$

Therefore, Eq. (17) is an algorithm for fusing the amplitude images with the weight coefficients $a_{\text {neg,pos }}^{\psi}, b_{n e g, p o s}^{\psi}$ and $c_{n e g, p o s}^{\psi}$. Regarding Eq. (21), the weight coefficients should not exceed unity, such that $a_{n e g, p o s}^{\psi}$ and $b_{n e g, p o s}^{\psi}$ are independent, whereas $c_{n e g, p o s}^{\psi}$ is determined as 


$$
c_{\text {neg }, \text { pos }}^{\psi}=\sqrt{1-\left(\left(a_{\text {neg, pos }}^{\psi}\right)^{2}+\left(b_{\text {neg }, \text { pos }}^{\psi}\right)^{2}\right)} .
$$

\subsubsection{Particular cases of normalization}

$\underline{\text { The case }} c_{\text {neg,pos }}^{\psi}=0 . \underline{\text { Weighted scalar complex-amplitude algorithm }}$

Under condition $\left(a_{\text {neg,pos }}^{\psi}\right)^{2}+\left(b_{\text {neg,pos }}^{\psi}\right)^{2}=1$, one has $c_{\text {neg,pos }}^{\psi}=0$ from Eq. (21), so that the amplitude of the fused image vector given by Eq. (17) reduces to the amplitude $\left|\vec{\psi}_{n e g, p o s}\right|^{2}=\left(a_{\text {neg, pos }}^{\psi} u\right)^{2}+\left(b_{\text {neg,pos }}^{\psi} v\right)^{2}$ of the weighted complex scalar functions $[13,14]$. Moreover, under the condition $\left(a_{\text {neg, pos }}^{\psi}\right)^{2}=\left(b_{\text {neg, pos }}^{\psi}\right)^{2}=1 / 2$, Eq. (21) reduces to the normalized amplitude algorithm $|\psi|^{n}=(1 / \sqrt{2}) \sqrt{u^{2}+v^{2}}$ (see Eq. (14) in Ref. [14]), which is based on the complex scalar functions $\psi_{n e g}=u+i v$ and $\psi_{p o s}=v+i u$. Therefore, we are led to conclude that the appropriate choice of the weight coefficients $a_{n e g, p o s}^{\psi}, b_{n e g, p o s}^{\psi}$ and $c_{n e g, p o s}^{\psi}$ (which, in their turn, are defined by the components of the transformation matrix $J$ via Eq. (18)) reduces the vector amplitude algorithm (17) of the CVIF method to the scalar amplitude algorithm of the SCFIF method $[13,14]$. The latter implies that the SCFIF method represents a particular case of the CVIF method.

$\underline{\text { The case }}\left(c_{\text {neg, pos }}^{\psi}\right)^{2}=2 a_{\text {neg, pos }}^{\psi} b_{\text {neg, pos }}^{\psi} \cdot \underline{\text { Simple scalar-addition algorithm }}$

Substitution of the condition $\left(c_{\text {neg, pos }}^{\psi}\right)^{2}=2 a_{\text {neg, pos }}^{\psi} b_{\text {neg,pos }}^{\psi}$ into Eq. (21) leads to the condition $a_{n e g, p o s}^{\psi}+b_{n e g, p o s}^{\psi}=1$. Then Eq. (17) reduces to the algorithm of weighted addition $|\psi|=a u+b v$ within the field of real scalars. In particular, Eq. (17) reduces to the arithmetic averaging algorithm $|\psi|=(u+v) / 2$ within the field of real scalars in the case of $a_{n e g, p o s}^{\psi}=b_{n e g, p o s}^{\psi}=1 / 2$ and at $c_{\text {neg,pos }}^{\psi}=2 a_{\text {neg,pos }}^{\psi} b_{\text {neg,pos }}^{\psi}$. In other words, at the appropriate choice of the weight coefficients $a_{n e g, p o s}^{\psi}, b_{n e g, p o s}^{\psi}$ and $c_{n e g, p o s}^{\psi}$ (which are defined by the components of the transformation matrix $J$ via Eq. (18)) the vector amplitude algorithm, which is defined by Eq. (17) within the field of complex vectors, reduces to the weighted addition algorithm and the averaging algorithms within the field of real scalars. In other terms, the action of the transformation matrix $J$ via Eq. (15) in case of the amplitude algorithm reduces to introduction of the weighted coefficients via Eq. (17).

\subsection{Ingredients of azimuth and ellipticity algorithms}

Let us find the azimuth and the ellipticity of the complex vector images $\vec{\psi}_{n e g}$,pos given by Eqs. (16). They are obtained by the action of matrix $J$ (see Eq. (14)) on the input complex vector image $\vec{\psi}_{\text {neg,pos }}^{(0)}$ given by Eqs. (1) and (2). For this aim one has to calculate the parameter

$$
t_{\text {neg, }, \text { pos }}=\frac{\psi_{2, \text { neg }, \text { pos }}}{\psi_{1, \text { neg, pos }}} \text {. }
$$

Here $\psi_{1, \text { neg,pos }}$ and $\psi_{2, \text { neg,pos }}$ are the components of the vector $\vec{\psi}_{\text {neg,pos }}$, which are defined respectively by the $1^{\text {st }}$ and $2^{\text {nd }}$ rows of the column vector given by Eqs. (16): 


$$
\begin{aligned}
& \psi_{1, \text { neg }}=\left(J_{11}^{\prime} u-J_{12}^{\prime \prime} v\right)+i\left(J_{11}^{\prime \prime} u+J_{12}^{\prime} v\right), \quad \psi_{1, \text { pos }}=\left(J_{11}^{\prime} v-J_{12}^{\prime \prime} u\right)+i\left(J_{11}^{\prime \prime} v+J_{12}^{\prime} u\right), \\
& \psi_{2, \text { neg }}=\left(J_{21}^{\prime} u-J_{22}^{\prime \prime} v\right)+i\left(J_{21}^{\prime \prime} u+J_{22}^{\prime} v\right), \quad \psi_{2, \text { pos }}=\left(J_{21}^{\prime} v-J_{22}^{\prime \prime} u\right)+i\left(J_{21}^{\prime \prime} v+J_{22}^{\prime} u\right) \text {. }
\end{aligned}
$$

Substituting Eqs. (24) into Eq. (23) gives

where

$$
t_{\text {neg,pos }}=\left.\frac{a+b d+i(b c-a d)}{\left(c^{2}+d^{2}\right)}\right|_{n e g, p o s}, \quad t_{n e g, p o s}^{*}=\left.\frac{a+b d-i(b c-a d)}{\left(c^{2}+d^{2}\right)}\right|_{n e g, p o s},
$$

From Eq. (25) one obtains

$$
\begin{array}{ll}
a_{\text {neg }}=J_{21}^{\prime} u-J_{22}^{\prime \prime} v, & a_{\text {pos }}=J_{21}^{\prime} v-J_{22}^{\prime \prime} u, \\
b_{\text {neg }}=J_{21}^{\prime \prime} u+J_{22}^{\prime} v, & b_{\text {pos }}=J_{21}^{\prime \prime} v+J_{22}^{\prime} u, \\
c_{\text {neg }}=J_{11}^{\prime} u-J_{12}^{\prime \prime} v, & c_{\text {pos }}=J_{11}^{\prime} v-J_{12}^{\prime \prime} u, \\
d_{\text {neg }}=J_{11}^{\prime \prime} u+J_{12}^{\prime} v, & d_{\text {pos }}=J_{11}^{\prime \prime} v+J_{12}^{\prime} u .
\end{array}
$$

$$
\begin{gathered}
\left|t_{\text {neg,pos }}\right|^{2}=\left.\frac{a^{2}+b^{2}}{c^{2}+d^{2}}\right|_{n e g, p o s}=\frac{\left|J_{21}\right|^{2} u^{2}+\left|J_{22}\right|^{2} v^{2}+2\left(J_{21}^{\prime \prime} J_{22}^{\prime}-J_{21}^{\prime} J_{22}^{\prime \prime}\right) u v}{\left|J_{11}\right|^{2} u^{2}+\left|J_{12}\right|^{2} v^{2}+2\left(J_{11}^{\prime \prime} J_{12}^{\prime}-J_{11}^{\prime} J_{12}^{\prime \prime}\right) u v} \\
t_{\text {neg, pos }}+t_{\text {neg, pos }}^{*}=\left.2 \frac{a c+b d}{c^{2}+d^{2}}\right|_{\text {neg,pos }}, \\
t_{\text {neg,pos }}-t_{\text {neg, pos }}^{*}=\left.2 i \frac{b c-a d}{c^{2}+d^{2}}\right|_{\text {neg,pos }}
\end{gathered}
$$

\subsection{Azimuth algorithms}

The azimuth is given by the formula

$$
\tan 2 \chi_{\text {neg, pos }}=\frac{t_{\text {neg, }, p o s}+t_{\text {neg, pos }}^{*}}{1-\left|t_{\text {neg, }, p o s}\right|^{2}} .
$$

Substituting Eqs. (25)-(29) into Eq. (30) yields in

$$
\tan 2 \chi_{\text {neg, pos }}=\left.2 \frac{a c+b d}{c^{2}+d^{2}-\left(a^{2}+b^{2}\right)}\right|_{\text {neg,pos }} .
$$

Eq. (31) can be rewritten as an explicit function of the input images $u$ and $v$ :

where

$$
\tan 2 \chi_{n e g, p o s}=\left.\frac{\xi_{\chi n}^{2} u^{2}+\mu_{\chi n}^{2} v^{2}+\sigma_{\chi n}^{2} u v}{\xi_{\chi d}^{2} u^{2}+\mu_{\chi d}^{2} v^{2}+\sigma_{\chi d}^{2} u v}\right|_{n e g, p o s},
$$

$$
\begin{aligned}
\xi_{\chi n, \text { neg }}^{2}=J_{21}^{\prime} J_{11}^{\prime}-J_{21}^{\prime \prime} J_{11}^{\prime \prime}, & \xi_{\chi d, \text { neg }}^{2}=\left|J_{11}\right|^{2}-\left|J_{21}\right|^{2}, \\
\mu_{\chi n, \text { neg }}^{2}=J_{22}^{\prime \prime} J_{12}^{\prime \prime}+J_{22}^{\prime} J_{12}^{\prime}, & \mu_{\chi d, \text { neg }}^{2}=\left|J_{12}\right|^{2}-\left|J_{22}\right|^{2}, \\
\sigma_{\chi n, \text { neg }}^{2}=J_{21}^{\prime \prime} J_{12}^{\prime}-J_{21}^{\prime} J_{12}^{\prime \prime}+J_{22}^{\prime} J_{11}^{\prime \prime}-J_{22}^{\prime \prime} J_{11}^{\prime}, & \sigma_{\chi d, \text { neg }}^{2}=2\left(J_{11}^{\prime \prime} J_{12}^{\prime}-J_{11}^{\prime} J_{12}^{\prime \prime}+J_{21}^{\prime} J_{22}^{\prime \prime}-J_{21}^{\prime \prime} J_{22}^{\prime}\right), \\
\xi_{\chi n, \text { pos }}^{2}=\mu_{\chi n, \text { neg }}^{2}, & \xi_{\chi d, \text { pos }}^{2}=\mu_{\chi d, \text { neg }}^{2}, \\
\mu_{\chi n, \text { pos }}^{2}=\xi_{\chi n, \text { neg }}^{2}, & \mu_{\chi d, \text { pos }}^{2}=\xi_{\chi d, \text { neg }}^{2}, \\
\sigma_{\chi n, \text { pos }}^{2}=\sigma_{\chi n, \text { neg }}^{2}, & \sigma_{\chi d, p o s}^{2}=\sigma_{\chi d, \text { neg }}^{2} .
\end{aligned}
$$




\subsection{Ellipticity algorithms}

The ellipticity is given by

$$
\sin 2 \gamma_{\text {neg,pos }}=-i \frac{t_{\text {neg, }, p o s}-t_{\text {neg }, p o s}^{*}}{1+\left|t_{\text {neg }, p o s}\right|^{2}} .
$$

Substituting Eqs. (25)-(29) into Eq. (35) results in

$$
\sin 2 \gamma_{\text {neg, pos }}=\left.2 \frac{b c-a d}{a^{2}+b^{2}+c^{2}+d^{2}}\right|_{\text {neg,pos }} .
$$

We rewrite Eq. (36) as a function of the input images $u$ and $v$ :

$$
\sin 2 \gamma_{n e g, p o s}=\left.\frac{\xi_{\gamma n}^{2} u^{2}+\mu_{\gamma n}^{2} v^{2}+\sigma_{\gamma n}^{2} u v}{\xi_{\gamma d}^{2} u^{2}+\mu_{\gamma d}^{2} v^{2}+\sigma_{\gamma d}^{2} u v}\right|_{n e g, p o s},
$$

where

$$
\begin{array}{cc}
\xi_{\gamma n, \text { neg }}^{2}=J_{21}^{\prime \prime} J_{11}^{\prime}-J_{21}^{\prime} J_{11}^{\prime \prime}, & \xi_{\gamma d, \text { neg }}^{2}=\left|J_{11}\right|^{2}+\left|J_{21}\right|^{2}, \\
\mu_{\gamma n, \text { neg }}^{2}=J_{22}^{\prime \prime} J_{12}^{\prime}-J_{22}^{\prime} J_{12}^{\prime \prime}, & \mu_{\gamma d, \text { neg }}^{2}=\left|J_{12}\right|^{2}+\left|J_{22}\right|^{2}, \\
\sigma_{\gamma n, \text { neg }}^{2}=J_{22}^{\prime} J_{11}^{\prime}+J_{22}^{\prime \prime} J_{11}^{\prime \prime}-J_{21}^{\prime} J_{12}^{\prime}-J_{21}^{\prime \prime} J_{11}^{\prime \prime}, & \sigma_{\gamma d, \text { neg }}^{2}=2\left(J_{21}^{\prime \prime} J_{22}^{\prime}+J_{11}^{\prime \prime} J_{12}^{\prime}-J_{21}^{\prime} J_{22}^{\prime \prime}-J_{11}^{\prime} J_{12}^{\prime \prime}\right), \\
\xi_{\gamma n, \text { pos }}^{2}=\mu_{\gamma n, \text { neg }}^{2}, & \xi_{\gamma d, \text { pos }}^{2}=\mu_{\gamma d, \text { neg }}^{2}, \\
\mu_{\gamma n, \text { pos }}^{2}=\xi_{\gamma n, \text { neg }}^{2}, & \mu_{\gamma d, \text { pos }}^{2}=\xi_{\gamma d, \text { neg }}^{2}, \\
\sigma_{\gamma n, \text { pos }}^{2}=\sigma_{\gamma n, \text { neg }}^{2}, & \sigma_{\gamma d, \text { pos }}^{2}=\sigma_{\gamma d, \text { neg }}^{2} .
\end{array}
$$

\section{Discussion}

It is well known [15] that variation of the Jones-matrix components results in varying polarization state of the light wave emergent from optically anisotropic medium. These transformations of the emergent light can be consistently considered using the Poincare sphere. Due to the analogy of the Jones vector for light and the complex vector $\vec{\psi}$ for fused image, the same approach can be applied to the image fusion performed with the CVIF method. Application of the Poincare sphere to the problems of image fusion is an interesting problem for the future work, which deserves further investigation.

It would be interesting to compare the vector fusion method suggested in this work with the recent scalar SCFIF method [13, 14]. In the framework of the SCFIF method [13, 14], one of the partial images is chosen as a real part and the other as an imaginary part of a complex function, which is then considered as a complex scalar fused image and is treated according to the rules of complex scalar function calculus. The SCFIF method in the field of complex scalars is an analogue of the fusion algorithm based on the operation of addition in the field of real scalars, although the partial images are not added directly in the framework of the SCFIF method. Within the field of complex scalar numbers, one can generate two principally different types of the fused images from the two partial images, the amplitude and phase ones. By its physical sense, the amplitude image can be understood as a root-mean square of the two partial images. On the other hand, the phase image can be calculated either as the phase of complex function (i.e., the arctangent of the ratio of partial images, which corresponds to a so-called $\varphi$-algorithm) or the tangent of the phase itself, which is nothing but the ratio of the partial images (a so-called $t$-algorithm).

Ukr. J. Phys. Opt. 2021, Volume 22, Issue 3 
Since there is no restriction on which of the two partial images must be taken as a real part of a complex scalar function, each of the $\varphi$ - and $t$ - phase algorithms splits into two sub-algorithms of 'positive' and 'negative' phase images. Therefore, within the field of complex scalar numbers, one can generate from two partial images at least five different fused images, which should be compared with a single fusion algorithm of image addition performed in the field of real scalars. Moreover, the other possibilities for the synthesis of additional fusion algorithms are available according to the rules of complex-function calculus $[13,14]$.

The advantages of the SCFIF method, if compared to its counterpart based on the operation of addition in the field of real scalars, are numerous. They cannot be reduced solely to a number of possible fused images. First, both the amplitude and phase images carry specific information of their own. For this reason they should not be considered as alternatives to each other. Instead, they are mutually complementary in the same sense as the amplitude and phase of a complex number are.

Second, multiple partial images obtained with Vis and IR cameras in the framework of the SCFIF method can be sorted respectively into two sets. They are pre-fused separately within each of the two sets followed by their fusion according to the rules of complex scalar function calculus as the amplitude and phase images. Such a procedure allows one to resolve ambiguity in selecting the pairs of images and the order of their fusion. Third, the image-quality indices for the SCFIF algorithms are better than those for the real scalar-addition algorithm. The other advantages of generalization concerned with passing to the field of complex scalars have been discussed in detail in Refs. $[13,14]$. The same advantages are peculiar to the case when we pass to the complex vector field in the framework of our CVIF method.

Besides of these common advantages, the CVIF method offered by us has its own advantages if compared with its scalar counterpart, the SCFIF method. One of them is based on Eq. (17), according to which the amplitude images $\left|\vec{\psi}_{n e g, p o s}\right|$ contain the coefficients $a_{n e g, p o s}^{\psi}, b_{n e g, p o s}^{\psi}$ and $c_{n e g, p o s}^{\psi}$ normalized via Eq. (21), such that only two of them appear to be independent. If one chooses $a_{n e g, p o s}^{\psi}$ and $b_{n e g, p o s}^{\psi}$ to be independent, then the third one, $c_{n e g, p o s}^{\psi}$, can be calculated from Eq. (22).

It is important that the weight coefficients $a_{n e g, p o s}^{\psi}$ and $b_{n e g, p o s}^{\psi}$, and thereby $c_{n e g, p o s}^{\psi}$, can be varied smoothly. This possibility implies that the image fusion can be done in a dynamic mode via animating the fused images in the course of variation of the weight coefficients. To illustrate application of this theoretical approach, we have fused the two input images obtained with a conventional digital camera operating in the Vis spectral range (see Fig. 1a) and a thermal camera for the IR range (see Fig. 1b). The dynamic mode of observation of the fused image can be of great aid to operators seeking for a target. The illustration of dynamic fusion of the input Vis (see Fig. 1a) and IR (see Fig. 1b) images with the amplitude algorithm,

$$
|\psi|=\sqrt{(a u)^{2}+(b v)^{2}+\left(1-a^{2}-b^{2}\right) u v},
$$

is presented in Fig. 2c. Note that Eq. (40) has been obtained by substituting Eq. (22) into Eq. (17).

Notice that a person on right, which cannot be seen in the Vis image (see Fig. 1a) becomes visible on the IR-image (see Fig. 1b). The fusion of the Vis and IR images combines the features of the both images in a single fused image. The images fused with the amplitude algorithm given by Eq. (40) in the stationary mode with the coefficients $a=0.5, b=0$ and $a=0.5, b=0.5$ are 
shown in Fig. 2a and Fig. 2b, respectively. A person on right, which cannot be observed in Fig. 1a becomes visible in the both images of Fig. $2 \mathrm{a}$ and Fig. $2 \mathrm{~b}$. The fused images obtained at different coefficients $a$ and $b$ shown in Fig. 2a and Fig. 2b differ somewhat by their contrasts, though not too much. It would be hard for an operator to decide which of the images obtained at these $a$ and $b$ pairs is more informative.

By varying the coefficients $a \in[1 ; 0]$ and $b \in[0 ; 1]$, one can transform smoothly the fused image and then compose the obtained set of resulting fused images into a video file (see Fig. 2c). Observation of the changes occurring with the fused image as a movie in the course of variation of the weight coefficients helps operator to catch targeting features. This is performed without
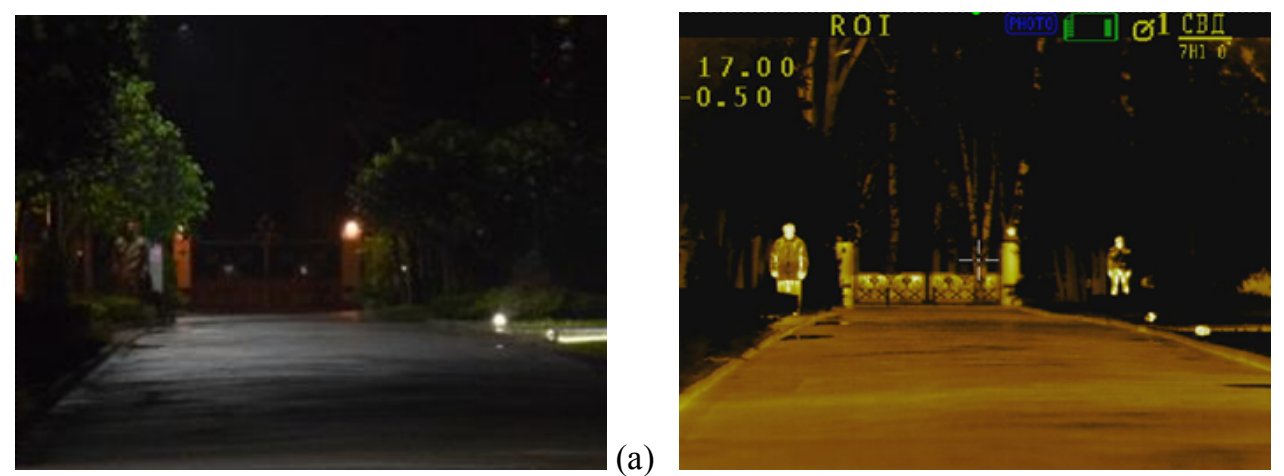

Fig. 1. Input images obtained using (a) a digital photo-camera in the Vis range and (b) thermal camera in the IR range.
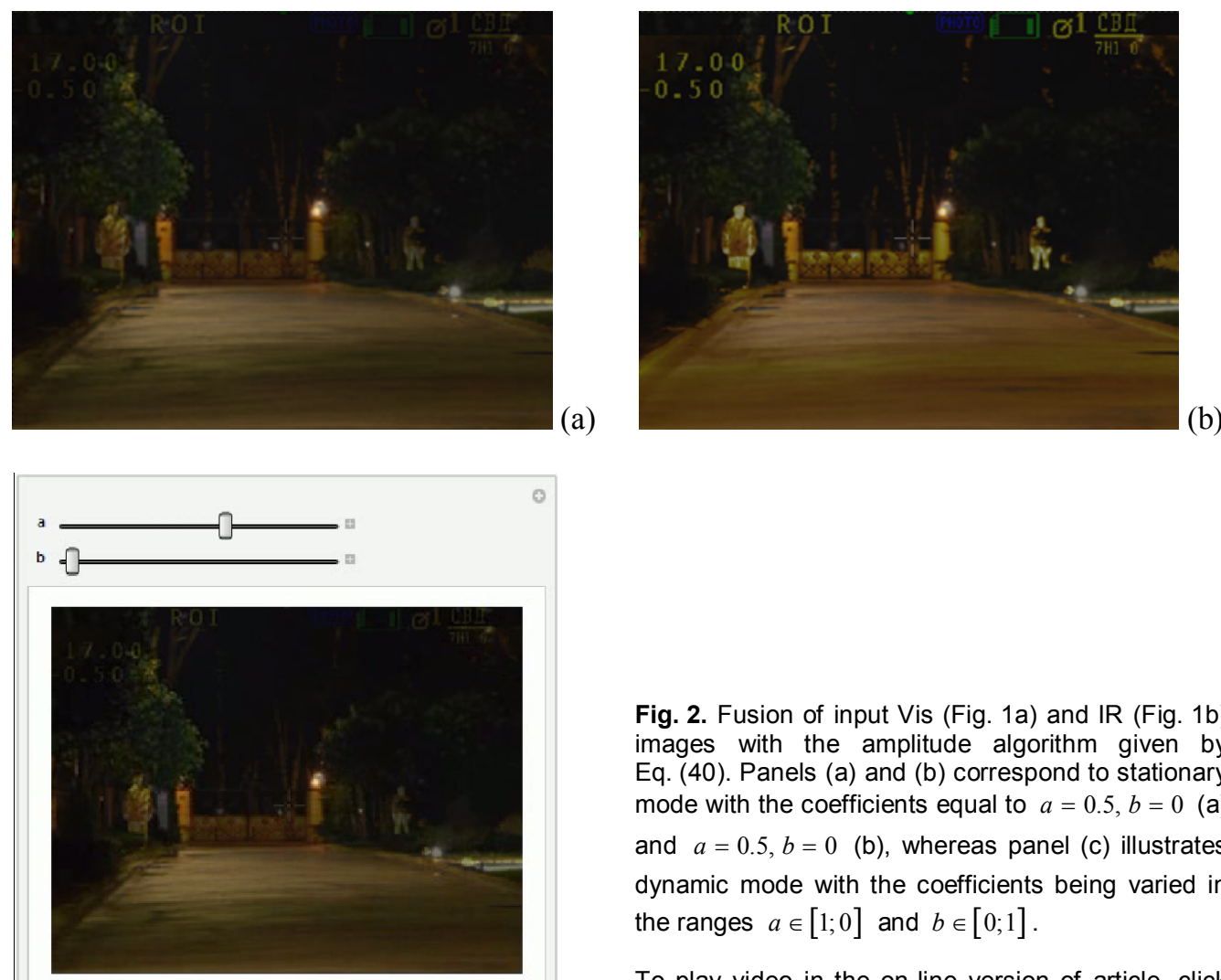

Fig. 2. Fusion of input Vis (Fig. 1a) and IR (Fig. 1b) images with the amplitude algorithm given by Eq. (40). Panels (a) and (b) correspond to stationary mode with the coefficients equal to $a=0.5, b=0$ (a) and $a=0.5, b=0$ (b), whereas panel (c) illustrates dynamic mode with the coefficients being varied in the ranges $a \in[1 ; 0]$ and $b \in[0 ; 1]$.

To play video in the on-line version of article, click (c) on the image.

Ukr. J. Phys. Opt. 2021, Volume 22, Issue 3 
focusing separately on each intermediate fused image, switching between them and comparing them, and choosing which of the fused images is better. Instead, the movie can be simply stopped by operator at the best visibility of a targeted feature. The image changing in time is also more convenient for computer-assisted targeting, including target detection, recognition and tracking. The dynamic mode of the image fusion suggested above enhances the probability of detection of target features (including a person on right) due to smooth variation of the fused image. It is known that human eyes perceive better and react more reliably to the image features which change in time. A periodical change helps catching reliably a target (by either operator or a computer). It is especially true if a target is absent on the image at some weight coefficients and then smoothly comes into view in the course of their variation, as illustrated in the video displayed in Fig. 2c.

It should be noted that the equations derived for the amplitude image-fusion algorithms given by Eq. (17) contain variables with the subscripts "neg" and "pos". The negative (neg) amplitude image can be transformed into the positive one, and vice versa, by interchanging the coefficients $a_{n e g, p o s}^{\psi}$ and $b_{n e g, p o s}^{\psi}$ involved in Eqs. (17) and (22). In the dynamic fusion mode, when these coefficients become nothing else but the weight coefficients which run from 0 to 1 and back again, the pos-image transforms into neg-image and back again. Hence, there is no need to keep longer these subscripts in Eq. (40).

The dynamic mode of the image fusion can also be applied to the azimuth (see Eq. (32)) and ellipticity (see Eq. (37)) algorithms in the same manner, as it has been demonstrated for the amplitude algorithm. This will be a subject of our future work.

\section{Conclusions}

We have presented a novel approach to the image fusion on the pixel level, which is based on the Jones formalism developed originally for the propagation of polarized light through optically anisotropic media [15]. Our approach is possible due to analogy between the Jones vectors for an elliptically polarized light wave and the complex vectors $\vec{\psi}_{\text {neg,pos }}^{0}$ composed of Vis $(u)$ and IR $(v)$ input images, according to Eqs. (1) and (2). In the framework of this analogy, one finds a vector $\vec{\psi}_{\text {neg,pos }}=J \vec{\psi}_{\text {neg,pos }}^{0}$ transformed by a complex $2 \times 2$-matrix $J$, an analogue of the Jones matrix for optically anisotropic medium. Basing on this analogy, one can compose three types of the fusion algorithms called as amplitude, azimuth and ellipticity ones.

Since the coefficients referred to the input images $u$ and $v$ in the frame of amplitude, azimuth and ellipticity algorithms can be smoothly varied, we offer a dynamic image-fusion method. The latter enables observing a fused image in a video format by varying these coefficients, which are combinations of the components of Jones matrix $J$. It is clear that a dynamic mode of observation that corresponds to changing the fused image in time enhances the probability of detecting a target, if compared with a common case of stationary image.

\section{References}

1. Mitchell H B, Image Fusion: Theories, Techniques and Applications. Berlin, Heidelberg: SpringerVerlag (2010).

2. Li S, Kang X and Hu J, 2013. Image fusion with guided filtering. IEEE Trans. Image Process. 22: 2864-2875.

3. Yang B and Li S, 2014. Visual attention guided image fusion with sparse representation. Optik. 125: $4881-4888$. 
4. Liu Y, Liu S and Wang Z, 2015. A general framework for image fusion based on multi-scale transform and sparse representation. Inf. Fusion. 24: 147-164.

5. Bavirisetti D P, Xiao G and Liu G. Multi-sensor image fusion based on fourth order partial differential equations. In: International Conference on Information Fusion, 2017, pp. 1-9.

6. Bavirisetti D P and Dhuli R, 2016. Two-scale image fusion of visible and infrared images using saliency detection. Infrared Phys. Technol. 76: 52-64.

7. Xiao G, Bavirisetti D P, Liu G and Zhang X. Image fusion based on machine learning and deep learning. In: Image Fusion. Springer, Singapore, 2020, pp. 325-352.

8. Yu Liu, Xun Chen, Zengfu Wang, Z Jane Wang, Raba K Ward and Xuesong Wang. 2018. Deep learning for pixel-level image fusion: Recent advances and future prospects. Inform. Fusion. 42: $158-173$.

9. Jingchun Piao, Yunfan Chen and Hyunchul Shin, 2019. A new deep learning based multi-spectral image fusion method. Entropy. 21: 570.

10. Yu Zhang, Yu Liu, Peng Sun, Han Yan, Xiaolin Zhao and Li Zhang, 2020. IFCNN: A general image fusion framework based on convolutional neural network. Inform. Fusion. 54: 99-118.

11.Kong W, Zhang L and Lei Y, 2014. Novel fusion method for visible light and infrared images based on nsst-sf-pcnn. Infrared Phys. Technol. 65: 103-112.

12. Sreeja $G$ and Saraniya O, 2019. Chapter 3. Image fusion through deep convolutional neural network. In Deep learning and parallel computing environment for bioengineering systems. pp. 37-52.

13. Khaustov Ya Ye, Khaustov D Ye, Ryzhov Ye, Lychkovskyy E and Nastishin Yu A, 2020. Fusion of visible and infrared images via complex function. Military Techn. Collection. 22: 20-31.

14. Khaustov Ya Ye, Khaustov D Ye, Hryvachevskyi A P, Ryzhov Ye, Lychkovskyy E, Prudyus I N and Nastishin Yu A, 2021. Complex function as a template for image fusion. Results in Opt. 2: 100038 .

15. Azzam R M A and Bashara N M. Ellipsometry and Polarized Light. New York: North-Holland Amsterdam (1977).

16. Nastyshyn S Yu, Bolesta I M, Tsybulia S A, Lychkovskyy E, Yakovlev M Yu, Ryzhov Ye, Vankevych P I and Nastishin Yu A, 2018. Differential and integral Jones matrices for a cholesteric. Phys. Rev. A. 97: 053804.

17. Nastyshyn S Yu, Bolesta I M, Tsybulia S A, Lychkovskyy E, Fedorovych Z Ya, Khaustov D Ye, Ryzhov Ye, Vankevych P I and Nastishin Yu A, 2019. Optical spatial dispersion in terms of Jones calculus. Phys. Rev. A. 100: 013806.

18. Konstantinova A F, Grechushnikov B N, Bokut B V and Valyashko Ye G. Optical Properties of Crystals. Minsk: Navuka i Teknnika (1995).

19. Khaustov Ya Ye, Khaustov D Ye, Lychkovskyy E, Ryzhov Ye and Nastishin Yu A, 2019. Image fusion for a target sightseeing system of armored vehicles. Military Techn. Collection. 21: 28-37.

20.Kurik M V and Lavrentovich O D, 1982. Negative-positive monopole transitions in cholesteric liquid crystals. JETP Lett. 35: 444-447.

21.Dirac P, 1931. Quantised singularities in the electromagnetic field. Proc. Roy. Soc. A. 133: 60-72.

22. Maxwell J C. In: The Scientific Papers of James Clerk Maxwell, Ed, by W D Niven. Cambridge: Cambridge University Press (1890).

23. Podolefsky N S and Finkelstein N D, 2006. Use of analogy in learning physics: The role of representations. Phys. Rev. ST Phys. Educ. Res. 2: 020101.

24.Feynman R P, 1957. Superfluidity and superconductivity. Rev. Mod. Phys. 29: 205-212.

Ukr. J. Phys. Opt. 2021, Volume 22, Issue 3 
25. de Gennes P G, 1972. An analogy between superconductors and smectics A. Solid State Commun. 10: $753-756$.

26.Renn S R and Lubensky T C, 1988. Abrikosov dislocation lattice in a model of the cholesteric-tosmectic-A transition. Phys. Rev. A. 38: 2132-2147.

27. Goodby J W, Waugh M A, Stein S M, Chin E, Pindak R and Patel J S, 1989. Characterization of a new helical smectic liquid crystal. Nature. 337: 449-452.

28.Lavrentovich O D, Nastishin Yu A, Kulishov V I, Narkevich Yu S, Tolochko A S and Shianovskii S V, 1990. Helical smectic A. Europhys. Lett. 13: 313-318.

29. Nguyen H T, Bouchta A, Navailles L, Barois P, Isaert N, Tweig R J, Maaroufi A and Destrade C, 1992. J. Phys. (Paris) II. 2: 1889-1906.

30.Pansu B, Nastishin Y, Imperor-Clerc M, Veber M and Nguyen H T, 2004. New investigations on the tetragonal liquid crystalline phase or SmQ. Eur. Phys. J. E. 15: 225-230.

Khaustov D. Ye., Khaustov Ya. Ye., Ryzhov Ye., Lychkowskyy E., Vlokh R. and Nastishin Yu. A. 2021. Jones formalism for image fusion. Ukr.J.Phys.Opt. 22: 165 - 180.

doi: $10.3116 / 16091833 / 22 / 3 / 165 / 2021$

Анотація. Запропоновано новий підхід до злиття видимих (u) та інфрачервоних (v) зображень, заснований на аналогії між математичними формами вектора Джонса для еліптично поляризованої світлової хвилі та комплексного двовимірного вектора $\vec{\psi}^{0}$, шо описує зображення и $i \mathrm{v}$. Оскільки немає обмежень стосовно того, яке з двох зображень слід обрати дійсною (або уявною) складовою, можна побудувати вектор $\vec{\psi}^{0}$ у двох формах - $\vec{\psi}_{\text {neg }}^{0}=(1 / \sqrt{2})[u, i v]^{T r}$ або $\vec{\psi}_{\text {pos }}^{0}=(1 / \sqrt{2})[v, i u]^{T r}$. Тут верхній індекс "Tr" позначае операцію транспонування, тобто $\vec{\psi}^{0}$ представляє собою вектор-стовпеиь. За аналогією $з$ вектором Джонса світлової хвилі, вектор $\vec{\psi}_{\text {pos,neg }}^{0}$ можна перетворювати згідно зі співвідноченням $\vec{\psi}=J \vec{\psi}_{\text {neg, роs }}^{0}$, де J представляє собою комплексну $2 \times 2$-матрицю, яка $\epsilon$ аналогом матрищі Джонса для оптично анізотропного середовища. Вищенаведена аналогія з джонсівським формалізмом дає змогу синтезувати злиті зображення, використовуючи три типи алгоритмів синтезу - «амплітудний», «азимутальний» $i$ «еліптичний». Змінюючи компоненти матриці $J$ з часом, можна синтезувати злите зображення в динамічному режимі, тим самим анімуючи зображення, які злиті згідно із параметрами, що $є$ комбінаціями компонентів матриці J і плавно змінюються в часі. 